\title{
Mesh erosion into bladder after transobturator prolapse repair
}

\author{
Folke Flam • Menachem Alcalay
}

Received: 5 August 2008 / Accepted: 18 September 2008 / Published online: 25 October 2008

(C) Springer-Verlag 2008

\begin{abstract}
We present three cases of mesh erosion into the bladder revealed from 5 to 36 months after cystocele repairs with Perigee ${ }^{\circledR}$ and Prolift $^{\circledR}$, respectively. The initial procedures were in two of the cases complicated by perforation of the bladder during the dissection. We describe different ways of treating this serious complication. Although these cases are anecdotal, it might be prudent not to proceed with the mesh procedure in cases of accidental opening of the bladder during the dissection.
\end{abstract}

Keywords Prolapse $\cdot$ Mesh $\cdot$ Bladder erosion

\section{Introduction}

In 2003, the Perigee ${ }^{\circledR}$ system (AMS, Minnetonka, MN, USA) and in 2004 Anterior Prolift ${ }^{\circledR}$ (Gynecare, Ethicon, Somerville, NJ, USA) were introduced for surgical use to reinforce the anterior vaginal wall. Needles are introduced from the skin through the obturator membranes in order to secure the mesh to the full length of the white line. These procedures seem to be easy to handle with few serious complications even during learning curves [1]. We have

Brief summary: three cases of late mesh erosions into the bladder following Perigee ${ }^{\circledR}$ and Prolift ${ }^{\circledR}$, respectively, are presented.

F. Flam $(\bowtie)$

Department of Gynecology, St Goran Hospital,

112 81, Stockholm, Sweden

e-mail: folke.flam@gynekologkliniken.se

M. Alcalay $(\bowtie)$

Department of Obstetrics and Gynecology,

Chaim Sheba Medical Center, Tel Aviv University,

52621, Tel Aviv, Israel

e-mail: malcalay@netvision.net.il learnt from non-mesh repairs that accidental tears into the bladder during the dissection are minor complications that will not affect outcome. It is not known if this is true also when a synthetic mesh is used in the repair. Mesh erosions into the bladder or urethra were reported following TVT and obturator slings [2,3]. Additionally, a few cases of bladder erosions following Perigee ${ }^{\circledR}$ or Prolift ${ }^{\circledR}$ have been published $[4,5]$. We present three cases of mesh erosion into the bladder that developed quite some time after the initial procedures and point to different strategies to restore bladder patency.

\section{Case reports}

1. A 52-year-old woman had within a few years time had two failed operations for a cystocele grade 2. While doing the Perigee ${ }^{\circledR}$, an inadvertent tear of the bladder of about $2 \mathrm{~cm}$ long was repaired in double layers. Three years later, cystoscopy revealed a small vesicovaginal fistula with just $2 \mathrm{~mm}$ of mesh eroded into the trigone. In the repair, a transabdominal approach was used where the visible part of the mesh as well as a small surrounding area was removed.

2. A 54-year-old woman had a recurrence of a cystocele after a conventional colporrhaphy. This time, anterior Prolift ${ }^{\circledR}$ was used for the grade 2 cystocele. Within hours, the woman complained of vaginal leakage and it was thought one of the arms possibly had perforated the bladder. However, at cystoscopy, irritation resembling cystitis was all that was seen. The leakage stopped on antibiotics. Two years later, a vesicovaginal fistula with mesh of $5 \mathrm{~mm}$ was diagnosed in the trigone. On top of this, bladder cancer was diagnosed in an area far away from the trigone. Histopathology 
revealed a TaG2-G3 cancer. On account of this, it was decided to perform a cystectomy and Bricker deviation.

3. A 52-year-old woman who had been hysterectomized experienced a grade 3 recurrence of a cystocele and a grade 2 recurrence of a rectocele. The plan was to implant total Prolift ${ }^{\circledR}$ along with a midurethral sling. During the operation, a bladder tear occurred. It was decided to proceed with the prolapse repair but not with the sling procedure. Three months later, the Monarch ${ }^{\circledR}$ (AMS) transobturator sling was successfully implanted. At this point, cystoscopy was normal. At follow-up 5 months after the Prolift ${ }^{\circledR}$ operation, the patient complained of urgency but not leakage. Supra-trigonal mesh erosion of $5 \mathrm{~mm}$ was diagnosed. Partial mesh excision was accomplished vaginally in the same way as a vesicovaginal fistula repair.

\section{Discussion}

We know from the TVT procedure that erosions into the bladder by the sling may occur years after the operations. In these instances, either an open abdominal surgery or a cystoscopic procedure will, for the most part, permanently take care of the problem. In the cases described in our report where a polypropylene mesh covers the whole surface of the descendent bladder, the clinician is faced with a more difficult problem. If only the exposed portion of the mesh is excised and the mesh was to erode again, the next operation would require a much larger excision leading potentially to a neobladder in the worst case scenario. This was indeed what happened in one of our cases but not due to mesh erosion but due to cancer by coincidence. On the other hand, one feels reluctant to excise as much mesh as possible causing unnecessary damage to the bladder. One of the three cases was treated through the vaginal route. Vesicovaginal fistulas following general gynecologic surgeries can successfully be repaired by this route but of course conditions are quite different when the underlying cause is a mesh. We suggest this route in cases where the eroded area is mobile enough. There probably is a better access and overview by doing the surgery from the abdomen but this should be weighed against the increased morbidity.

The good news about our three cases is that, retrospectively, none of them experienced uneventful primary surgeries. Two of them had bladder perforations while performing the dissection. The third patient had a marked inflammatory response seen at cystoscopy. In the latter case, no catheter had been used while performing the prolapse repair and it seems highly unlikely that the bladder appearance corresponded to a bacterial infection. It seems more likely that the mesh itself gave rise to this conditionmaybe because of a thin bladder wall. With time, more anterior repairs will be performed using non-absorbable mesh materials and we should expect more cases like the ones described. The authors have decided not to perform mesh repairs should an accidental tear of the bladder happen during the dissection. Most probably, this damage caused to the bladder is more severe than an accidental perforation of the bladder using the needles used in Perigee ${ }^{\circledR}$ and Prolift ${ }^{\circledR}$. At least this is the lesson we have learnt from TVT.

Conflict of interest Folke Flam is a trainer for Ethicon, Women's Health and Urology

\section{References}

1. Altman D, Falconer C (2007) Perioperative morbidity using transvaginal mesh in pelvic organ prolapse repair. Obstet Gynecol 109:303-308

2. Huang KH, Kung FT, Liang HM, Chang SY (2005) Management of polypropylene mesh erosion after intravaginal midurethral sling operation for female stress urinary incontinence. Int Urogynecol J Pelvic Floor Dysfunct 16:437-440

3. Parekh MH, Minassian VA, Poplawsky D (2006) Bilateral bladder erosion of a transobturator tape mesh. Obstet Gynecol 108:713-715

4. Yamada BS, Govier FE, Stefanovic KB, Kobashi KC (2006) Vesicovaginal fistula and mesh erosion after Perigee (transobturator polypropylene mesh anterior repair). Urology 68:1121-1124

5. Abdel-Fattah M, Ramsay I (2008) West of Scotland Study group. Retrospective multicentre study of minimal invasive mesh repair devices for pelvic organ prolapse. BJOG 115(1):22-30 